\title{
The Oppenheim series expansions and Hausdorff dimensions
}

\author{
by \\ Jun Wu (Wuhan)
}

1. Introduction. Any real number $x \in(0,1]$ has the Oppenheim expansion [14]

$$
x \sim \frac{1}{d_{1}}+\frac{a_{1}}{b_{1}} \frac{1}{d_{2}}+\ldots+\frac{a_{1} a_{2} \ldots a_{n}}{b_{1} b_{2} \ldots b_{n}} \frac{1}{d_{n+1}}+\ldots,
$$

where $a_{n}=a_{n}\left(d_{1}, \ldots, d_{n}\right), b_{n}=b_{n}\left(d_{1}, \ldots, d_{n}\right)$ are positive integers and the denominators $d_{n}$ are determined by the algorithm

$$
x=x_{1}, \quad d_{n}=\left[1 / x_{n}\right]+1, \quad x_{n}=1 / d_{n}+a_{n} / b_{n} \cdot x_{n+1}
$$

(here and in what follows $[y]$ denotes the integer part of $y$ ). By (2),

$$
\frac{1}{d_{n}}<x_{n} \leq \frac{1}{d_{n}-1}
$$

hence for any $n \geq 1$,

$$
d_{n+1}>\frac{a_{n}}{b_{n}} d_{n}\left(d_{n}-1\right) .
$$

The expansion defined by (1) and (2) is convergent and its sum is equal to $x$. A sufficient condition for a series on the right hand side in (1) to be the expansion of its sum by the algorithm (2) is (see [14])

$$
d_{n+1} \geq \frac{a_{n}}{b_{n}} d_{n}\left(d_{n}-1\right)+1 \quad \text { for any } n \geq 1 .
$$

DeFinition 1. We call the expansion (1) (obtained by the algorithm (2)) the restricted Oppenheim expansion of $x$ if $a_{n}$ and $b_{n}$ depend on the last denominator $d_{n}$ only and if the function

$$
h_{n}(j)=\frac{a_{n}(j)}{b_{n}(j)} j(j-1)
$$

is integer-valued. 
In the present paper, we deal with restricted Oppenheim expansions only. In this case, (4) and (5) are equivalent.

There has been much work on the metric theory of restricted Oppenheim expansions. One usually chooses Lebesgue measure on the Borel subsets of the interval $(0,1]$ as the underlying probability space, and asks about metric properties of digits in restricted Oppenheim expansions (see [1], [4][11], [15]-[18]). In this paper, we investigate the Hausdorff dimension of exceptional sets in the metric properties of digits in restricted Oppenheim expansions. The corresponding results for Engel and Sylvester expansions have been obtained by Liu and the author [12], [19]; here more attention is paid to the estimates in order to apply the mass distribution principle. On the way, we also consider the fractal dimension of the exceptional set of a uniformly distributed sequence modulo 1 .

We use $|\cdot|$ to denote the diameter of a subset of $(0,1], \operatorname{dim}_{H}$ to denote the Hausdorff dimension and $\mathrm{cl}$ the closure of a subset of $(0,1]$ respectively.

\section{Oppenheim series expansions and Hausdorff dimensions}

Definition 2. Let $t \geq 1$ and $\beta>0$. We say that the function $h_{n}(j)$ is of order $t$ with constant $\beta>0$ if

$$
\frac{h_{n}(j)}{j^{t}} \rightarrow \beta \quad \text { as } n, j \rightarrow \infty .
$$

We now list some special cases which have been considered in the literature earlier.

EXAMPLE 1 (Engel expansion). Let $a_{n}\left(d_{1}, \ldots, d_{n}\right)=1, b_{n}\left(d_{1}, \ldots, d_{n}\right)=$ $d_{n}(n=1,2, \ldots)$. Then (1), together with the algorithm (2), is the Engel expansion of $x$,

$$
x=\frac{1}{d_{1}}+\frac{1}{d_{1} d_{2}}+\ldots+\frac{1}{d_{1} d_{2} \ldots d_{n}}+\ldots
$$

Here $h_{n}(j)=j-1$ is of order 1 with $\beta=1$.

ExAmple 2 (Sylvester expansion). Choosing $a_{n}\left(d_{1}, \ldots, d_{n}\right)=1$, $b_{n}\left(d_{1}, \ldots, d_{n}\right)=1(n=1,2, \ldots)$, we get the Sylvester expansion of $x$,

$$
x=\frac{1}{d_{1}}+\frac{1}{d_{2}}+\ldots+\frac{1}{d_{n}}+\ldots
$$

In this case, $h_{n}(j)=j(j-1)$ is of order 2 with $\beta=1$.

ExAmple 3 (Generalized Cantor product). Taking $a_{n}\left(d_{1}, \ldots, d_{n}\right)=$ $k+d_{n}, b_{n}\left(d_{1}, \ldots, d_{n}\right)=d_{n}(n=1,2, \ldots)$, where $k$ is a fixed positive integer, yields the generalized Cantor product,

$$
1+k x=\left(1+\frac{k}{d_{1}}\right)\left(1+\frac{k}{d_{2}}\right) \ldots\left(1+\frac{k}{d_{n}}\right) \ldots
$$


Here $h_{n}(j)=(j+k)(j-1)$ is of order 2 with $\beta=1$. Generalized Cantor products were studied in Oppenheim [13]. For $k=2$, Escott [2] used the above product representation for a rapid approximation of quadratic irrationals.

ExAmple 4 (Modified Engel expansion). Letting $a_{n}\left(d_{1}, \ldots, d_{n}\right)=1$, $b_{n}\left(d_{1}, \ldots, d_{n}\right)=d_{n}-1(n=1,2, \ldots)$, we get the modified Engel expansion of $x$,

$$
x=\frac{1}{d_{1}}+\frac{1}{\left(d_{1}-1\right) d_{2}}+\ldots+\frac{1}{\left(d_{1}-1\right)\left(d_{2}-1\right) \ldots\left(d_{n-1}-1\right) d_{n}}+\ldots
$$

Thus $h_{n}(j)=j$ is of order 1 with $\beta=1$.

EXAmple 5 (Daróczy-Kátai-Birthday expansion). If we choose $a_{n}\left(d_{1}, \ldots, d_{n}\right)=d_{n}, b_{n}\left(d_{1}, \ldots, d_{n}\right)=1(n=1,2, \ldots)$, the resulting series expansion of $x$ takes the form

$$
x=\frac{1}{d_{1}}+\frac{d_{1}}{d_{2}}+\ldots+\frac{d_{1} d_{2} \ldots d_{n-1}}{d_{n}}+\ldots
$$

Here $h_{n}(j)=j^{2}(j-1)$ is of order 3 with $\beta=1$. This Daróczy-Kátai-Birthday expansion was introduced for the first time in Galambos [9].

All the expansions in the examples above are restricted Oppenheim expansions; note that in all these cases $h_{n}(j)$ is independent of $n$.

In this paper, we always assume $h_{n}(j)$ is of order $t(t \geq 1)$ with $\beta>0$. Under this assumption, for any $\varepsilon>0$, there exists a positive integer $n(\varepsilon)$ such that for any $n$ and $j \geq n(\varepsilon)$,

$$
h_{n}(j) \leq(\beta+\varepsilon) j^{t} .
$$

We first state the mass distribution principle (see [3, Proposition 2.3]) that will be used later.

Lemma 1. Let $E \subset(0,1]$ be a Borel set and $\mu$ be a measure with $\mu(E)>0$. If for any $x \in E$,

$$
\liminf _{r \rightarrow 0} \frac{\log \mu(B(x, r))}{\log r} \geq s
$$

where $B(x, r)$ denotes the open ball with center at $x$ and radius $r$, then $\operatorname{dim}_{\mathrm{H}} E \geq s$.

In what follows we often make use of a symbolic space defined as follows. Let $\left\{L_{n}: n \geq 1\right\}$ and $\left\{M_{n}: n \geq 1\right\}$ be two sequences of positive numbers such that $L_{1} \geq 1$ and $L_{k+1} \geq \max _{j}\left\{h_{k}(j):\left[L_{k}\right]+1 \leq j \leq\left[M_{k}\right]\right\}$ for any $k \geq 1$. For any $n \geq 1$, let

$$
D_{n}=\left\{\left(\sigma_{1}, \ldots, \sigma_{n}\right) \in \mathbb{N}^{n}: L_{k}<\sigma_{k} \leq M_{k} \text { for all } 1 \leq k \leq n\right\},
$$


and define

$$
D=\bigcup_{n=0}^{\infty} D_{n} \quad\left(D_{0}=\emptyset\right) .
$$

For any $\sigma=\left(\sigma_{1}, \ldots, \sigma_{n}\right) \in D_{n}$, let $J_{\sigma}$ denote the following closed subinterval of $(0,1]$ :

$$
J_{\sigma}=\operatorname{cl}\left\{x \in(0,1]: d_{1}(x)=\sigma_{1}, \ldots, d_{n}(x)=\sigma_{n}\right\} ;
$$

each $J_{\sigma}$ is called an interval of $n$th order. Define

$$
E=\bigcap_{n=1}^{\infty} \bigcup_{\sigma \in D_{n}} J_{\sigma} .
$$

It is obvious that

$$
E=\left\{x \in(0,1]: L_{n}<d_{n}(x) \leq M_{n} \text { for all } n \geq 1\right\} .
$$

For any $\alpha>0$, define

$$
A(\alpha)=\left\{x \in(0,1]: \lim _{n \rightarrow \infty} d_{n+1}(x) / d_{n}^{t}(x)=\alpha\right\} .
$$

TheOREM 1. If $h_{n}(j)$ is of order $t$ with $\beta>0$ and $t>1$, then

$$
\operatorname{dim}_{\mathrm{H}} A(\alpha)=1 \quad \text { for any } \alpha>\beta \text {. }
$$

Proof. We adopt the idea from [19]. Fix $\gamma$ such that $\beta<\gamma<\alpha$. By (13), there exists $n(\gamma)$ such that $h_{n}(j) \leq \gamma j^{t}$ for any $n$ and $j \geq n(\gamma)$. We consider three cases.

CASE 1: $\alpha>1$. Let $\left\{c_{n}: n \geq 1\right\}$ be a sequence such that $c_{1}=2$, $c_{n+1}=h_{n}\left(c_{n}\right)+2$ for any $n \geq 1$. Choose $n_{1} \geq n(\gamma)$ large enough such that for any $n \geq n_{1}$,

$$
\begin{gathered}
\left(\frac{n+1}{n}\right)^{t} \gamma<\alpha, \quad \frac{1}{n} \alpha^{t^{n}-1 /(t-1)}>1, \\
\alpha^{t^{n}-1 /(t-1)} \geq h_{n(\gamma)}\left(c_{n(\gamma)}\right)+1, \quad \alpha^{t^{n}-1 /(t-1)}>n(\gamma)+1 .
\end{gathered}
$$

For any $n \geq 1$, let

$$
\begin{array}{lll}
L_{n}=c_{n}-1 / 2, & M_{n}=c_{n}+1 / 2, & \text { for } 1 \leq n \leq n(\gamma), \\
L_{n}=\alpha^{t^{n+n_{1}}-1 /(t-1)}, & M_{n}=\frac{n+1}{n} \alpha^{t^{n+n_{1}}-1 /(t-1)}, & \text { for } n>n(\gamma) .
\end{array}
$$

Thus for any $n \geq n(\gamma)$ and $\left[L_{n}\right]+1 \leq j \leq\left[M_{n}\right]$, we have

$$
L_{n+1} \geq \alpha\left(\frac{n}{n+1}\right)^{t}\left[M_{n}\right]^{t}>\gamma\left[M_{n}\right]^{t} \geq \gamma j^{t}>h_{n}(j)
$$

this implies, for any $n>n(\gamma)$ and $\left[L_{n}\right]+1 \leq j \leq\left[M_{n}\right]$,

$$
\left[L_{n+1}\right]+1 \geq h_{n}(j)+1 \text {. }
$$


By the choice of $\left\{c_{n}: n \geq 1\right\}$ and the definition of $\left\{L_{n}: n \geq 1\right\},\left\{M_{n}: n \geq 1\right\}$, it is easy to see that formula (16) holds for any $n \geq 1$ and $\left[L_{n}\right]+1 \leq j \leq\left[M_{n}\right]$.

Now we estimate the length of $J_{\sigma}$ for any $\sigma \in D_{n}$. By [4, Lemma 1], we have

$\left|\left\{x \in(0,1]: d_{1}(x)=\sigma_{1}, \ldots, d_{n}(x)=\sigma_{n}\right\}\right|$

thus by (6),

$$
=\frac{a_{1}\left(\sigma_{1}\right) \ldots a_{n-1}\left(\sigma_{n-1}\right)}{b_{1}\left(\sigma_{1}\right) \ldots b_{n-1}\left(\sigma_{n-1}\right)} \cdot \frac{1}{\left(\sigma_{n}-1\right) \sigma_{n}},
$$

$$
c^{-n} \alpha^{-t^{n+n_{1}+1} /(t-1)} \leq\left|J_{\sigma}\right| \leq c^{n} \alpha^{-t^{n+n_{1}+1} /(t-1)},
$$

where $c$ is a positive constant which does not depend on $n$.

Let $\mu$ be a mass distribution supported on $E$ such that for any $n \geq 1$ and $\sigma \in D_{n}$,

$$
\mu\left(J_{\sigma}\right)=\frac{1}{\sharp D_{n}} \quad\left(\sharp D_{0}:=1\right),
$$

where $\sharp$ denotes the cardinality. By the definition of $D_{n}$, it is easy to check

$$
d^{-n} \alpha^{t^{n+n_{1}+1} /(t-1)} \leq \sharp D_{n} \leq d^{n} \alpha^{t^{n+n_{1}+1} /(t-1)},
$$

where $d$ is a positive constant which does not depend on $n$.

For any $x \in E$, we prove that

$$
\liminf _{r \rightarrow 0} \frac{\log \mu(B(x, r))}{\log r} \geq 1 .
$$

For $r<\alpha^{-t^{n_{1}+1} /(t-1)}$, choose a positive integer $k \geq t^{n_{1}+1} /(t-1)$ such that

$$
\alpha^{-k-1}<r \leq \alpha^{-k} \text {. }
$$

Choose $n_{k} \geq 1$ such that

and write

$$
\frac{t^{n_{k}+n_{1}}}{t-1} \leq k<\frac{t^{n_{k}+n_{1}+1}}{t-1}
$$

Then

$$
k=\frac{t^{n_{k}+n_{1}}}{t-1}+\widetilde{k}
$$

$$
\widetilde{k}<\frac{t^{n_{k}+n_{1}+1}}{t-1}-\frac{t^{n_{k}+n_{1}}}{t-1}=t^{n_{k}+n_{1}} .
$$

By inequalities (17) and (20), B(x,r) can intersect at most $4 c^{n_{k}} \alpha^{t^{n_{1}+n_{k}}-\widetilde{k}}$ $n_{k}$ th-order intervals, thus by (18) and (19),

$$
\begin{aligned}
& \liminf _{r \rightarrow 0} \frac{\log \mu(B(x, r))}{\log r} \\
& \quad \geq \liminf _{k \rightarrow \infty} \frac{\log \left(d^{n_{k}} \alpha^{-t^{n_{1}+n_{k}+1} /(t-1)} 4 c^{n_{k}} \alpha^{t^{n_{1}+n_{k}}-\widetilde{k}}\right)}{\log \alpha^{-t^{n_{1}+n_{k}} /(t-1)-\widetilde{k}-1}}=1 .
\end{aligned}
$$


By Lemma 1, we have $\operatorname{dim}_{\mathrm{H}} E=1$. To finish the proof of Case 1, it is enough to show $E \subset A(\alpha)$.

By the definition of $E,\left\{L_{n}: n \geq 1\right\}$ and $\left\{M_{n}: n \geq 1\right\}$, we have, for any $x \in E$ and $n>n(\gamma)$,

$$
\left(\frac{n}{n+1}\right)^{t} \alpha \leq \frac{d_{n+1}(x)}{d_{n}^{t}(x)} \leq \frac{n+2}{n+1} \alpha,
$$

therefore

$$
\lim _{n \rightarrow \infty} \frac{d_{n+1}(x)}{d_{n}^{t}(x)}=\alpha
$$

CASe 2: $\alpha=1$. As this case is very similar to Case 1 , we just give an outline. Let $\gamma, n(\gamma)$ and $\left\{c_{n}: n \geq 1\right\}$ be as in Case 1 , and choose $n_{1} \geq n(\gamma)$ large enough such that for any $n \geq n_{1}$,

$$
\begin{gathered}
\left(\frac{n+1}{n}\right)^{t} \gamma<1, \quad \frac{1}{n} e^{t^{n}}>1, \\
e^{t^{n} \geq h_{n(\gamma)}\left(c_{n(\gamma)}\right)+1,} \quad e^{t^{n}}>n(\gamma)+1 .
\end{gathered}
$$

For any $n \geq 1$, let

$$
\begin{array}{lll}
L_{n}=c_{n}-1 / 2, & M_{n}=c_{n}+1 / 2, & \text { for } 1 \leq n \leq n(\gamma), \\
L_{n}=e^{t^{n+n_{1}}}, & M_{n}=\frac{n+1}{n} e^{t^{n+n_{1}}}, & \text { for } n>n(\gamma) .
\end{array}
$$

Following the proof of Case 1 step by step, we get $\operatorname{dim}_{\mathrm{H}} A(1)=1$.

CASE 3: $\alpha<1$. In this case, $0<\beta<\alpha<1$. Let $\gamma, n(\gamma)$ and $\left\{c_{n}: n \geq 1\right\}$ be as in Case 1 , and choose $n_{1} \geq n(\gamma)$ large enough such that for any $n \geq n_{1}$,

$$
\begin{gathered}
\left(\frac{n+1}{n}\right)^{t} \gamma<\alpha, \quad \frac{1}{n} \alpha^{-t^{n}-1 /(t-1)}>1, \\
\alpha^{-t^{n}-1 /(t-1)} \geq h_{n(\gamma)}\left(c_{n(\gamma)}\right)+1, \quad \alpha^{-t^{n}-1 /(t-1)}>n(\gamma)+1 .
\end{gathered}
$$

For any $n \geq 1$, let

$$
\begin{array}{lll}
L_{n}=c_{n}-1 / 2, & M_{n}=c_{n}+1 / 2, & \text { for } 1 \leq n \leq n(\gamma), \\
L_{n}=\alpha^{-t^{n+n_{1}}-1 /(t-1)}, & M_{n}=\frac{n+1}{n} \alpha^{-t^{n+n_{1}}-1 /(t-1)}, & \text { for } n>n(\gamma) .
\end{array}
$$

Following the proof of Case 1 step by step, we again get $\operatorname{dim}_{\mathrm{H}} A(\alpha)=1$. The proof of Theorem 1 is finished.

Now we consider the case when $h_{n}(j)$ is of order 1 .

THEOREM 2. If $h_{n}(j)$ is of order 1 with $\beta>0$, then

$$
\operatorname{dim}_{\mathrm{H}} A(\alpha)=1 \quad \text { for any } \alpha>\max (1, \beta) .
$$


Proof. Let $\gamma, n(\gamma)$ and $\left\{c_{n}: n \geq 1\right\}$ be as in Theorem 1, and choose $n_{1} \geq n(\gamma)$ large enough such that for any $n \geq n_{1}$,

$$
\frac{n+1}{n} \gamma<\alpha, \quad \frac{1}{n} \alpha^{n}>1, \quad \alpha^{n} \geq h_{n(\gamma)}\left(c_{n(\gamma)}\right)+1, \quad \alpha^{n}>n(\gamma)+1 .
$$

For any $n \geq 1$, let

$$
\begin{array}{lll}
L_{n}=c_{n}-1 / 2, & M_{n}=c_{n}+1 / 2, & \text { for } 1 \leq n \leq n(\gamma), \\
L_{n}=\alpha^{n+n_{1}}, & M_{n}=\frac{n+1}{n} \alpha^{n+n_{1}}, & \text { for } n>n(\gamma) .
\end{array}
$$

Proceeding as in Theorem 1, we get the assertion.

Theorem 3. If $h_{n}(j)$ is of order 1 with $0<\beta<1$, then $\operatorname{dim}_{\mathrm{H}} A(1)=1$.

Proof. Let $\gamma, n(\gamma)$ and $\left\{c_{n}: n \geq 1\right\}$ be as in Theorem 1, and choose $n_{1} \geq n(\gamma)$ large enough such that for any $n \geq n_{1}$,

$$
\begin{gathered}
\left(\frac{1+1 / \sqrt{n+1}}{1+1 / \sqrt{n}}\right)^{n}>\gamma, \quad\left(1+\frac{1}{\sqrt{n}}\right)^{n}>1, \\
n\left(1+\frac{1}{\sqrt{n}}\right)^{n} \geq h_{n(\gamma)}\left(c_{n(\gamma)}\right)+1, \quad n\left(1+\frac{1}{\sqrt{n}}\right)^{n}>n(\gamma)+1 .
\end{gathered}
$$

For any $n \geq 1$, let

$$
\begin{gathered}
L_{n}=c_{n}-1 / 2, \quad M_{n}=c_{n}+1 / 2, \quad \text { for } 1 \leq n \leq n(\gamma), \\
L_{n}=n\left(1+\frac{1}{\sqrt{n+n_{1}}}\right)^{n+n_{1}}, \quad M_{n}=(n+1)\left(1+\frac{1}{\sqrt{n+n_{1}}}\right)^{n+n_{1}}, \quad \text { for } n>n(\gamma) .
\end{gathered}
$$

Just as in Theorem 1, we get $\operatorname{dim}_{\mathrm{H}} A(1)=1$.

REMARK 1 . When $h_{n}(j)$ is of order 1 with $0<\beta<1$, it is easy to see that for any $\beta<\alpha<1$, the set

$$
A(\alpha)=\left\{x \in(0,1]: \lim _{n \rightarrow \infty} d_{n+1}(x) / d_{n}(x)=\alpha\right\}
$$

is empty. Thus the conclusions of Theorems 2 and 3 cannot hold.

REMARK 2. Let $h_{n}(j)$ be of order $t$ with $\beta>0(\beta \geq 1$ when $t=1)$, and let $\alpha=\beta$. We do not know whether the conclusions of Theorems 1-3 hold when $\alpha=\beta$. In the next section, we show that the results are true when $h_{n}(j)$ is a polynomial.

By Theorems 1 and 2, we get the following corollaries immediately.

COROllary 1. If $h_{n}(j)$ is of order $t$ with $\beta>0$, then for any $M>$ $\max (1,1 / \beta)$, the set

$$
\bar{A}(M)=\left\{x \in(0,1]: \lim _{n \rightarrow \infty} \frac{h_{n}\left(d_{n}(x)\right)}{d_{n+1}(x)}=\frac{1}{M}\right\}
$$

is of Hausdorff dimension 1. 
Galambos [4] (see also [7]) showed that if $h_{n}(j) \geq j-1$ for all $n$, then, for almost all $x$, as $n \rightarrow \infty$,

$$
\frac{1}{n} \sum_{l=1}^{n} \log \frac{d_{l}(x)}{h_{l-1}\left(d_{l-1}(x)\right)} \rightarrow 1 \quad\left(h_{0}(k):=1\right) .
$$

Corollary 2. Suppose that $h_{n}(j) \geq j-1$ for all $n$ and $h_{n}(j)$ is of order $t$ with $\beta>0$ and $t \geq 1$. Then, for any $k>1$, the set

$$
\left\{x \in(0,1]: \sum_{l=1}^{n} \log \frac{d_{l}(x)}{h_{l-1}\left(d_{l-1}(x)\right)} \geq k n \text { for any } n \geq 1\right\}
$$

is of Hausdorff dimension 1. In particular, the Hausdorff dimension of the set where (21) fails is 1 .

3. The case of $h_{n}(j)$ being a polynomial. In this section, we shall discuss the cases when $h_{n}(j)$ is a polynomial for any $n$ and get a little stronger results than those in Theorems 1-3.

We first investigate the case when $h_{n}(j)$ is linear for any $n$.

THEOREM 4. If $h_{n}(j)=a j+b$ with $a>0$, then $\operatorname{dim}_{\mathrm{H}} A(\alpha)=1$ for any $\alpha \geq \max (1, a)$.

Proof. By Theorems 2 and 3, it is enough to deal with the case $\alpha=$ $a \geq 1$. Here we only investigate the situation when $\alpha=a>1$. The case $\alpha=a=1$ can be discussed in a similar way to Theorem 3 .

Choose $n_{1}$ large enough such that $\alpha^{n}>b+1$ for any $n \geq n_{1}$. For any $n \geq 1$, let

$$
L_{n}=\left(2\left(n+n_{1}\right)-1\right) \alpha^{n+n_{1}}, \quad M_{n}=2\left(n+n_{1}\right) \alpha^{n+n_{1}} .
$$

Then, for any $n \geq 1$ and $L_{n}<j<M_{n}$,

$$
L_{n+1}=\left(2\left(n+n_{1}+1\right)-1\right) \alpha^{n+n_{1}+1}>\alpha M_{n}+b+1 \geq h_{n}(j)+1 .
$$

Following the proof of Theorem 1, we get the assertion.

Corollary 3. For Engel and modified Engel expansions, for any $\alpha \geq 1$, the set

$$
\left\{x \in(0,1]: \lim _{n \rightarrow \infty} d_{n+1}(x) / d_{n}(x)=\alpha\right\}
$$

is of Hausdorff dimension 1 .

THEOREM 5. Suppose $h_{n}(j)=a_{m} j^{m}+a_{m-1} j^{m-1}+\ldots+a_{1} j+a_{0}$ with $a_{m}>0$ and $m \geq 2$. Then $\operatorname{dim}_{\mathrm{H}} A(\alpha)=1$ for any $\alpha \geq a_{m}$.

Proof. By Theorem 1, it is enough to deal with the case $\alpha=a_{m}$. As in Theorem 1, we can divide the proof into three cases: $\alpha=a_{m}>1, \alpha=$ $a_{m}=1$ and $\alpha=a_{m}<1$. Here we only investigate the case $\alpha=a_{m}>1$, 
the other two can be discussed similarly to Theorem 1. Define $\widetilde{h}_{n}(j)=$ $a_{m-1} j^{m-1}+\ldots+a_{1} j+a_{0}$ and

$$
K(n)=\max \left\{\widetilde{h}_{n}(k)+1: \alpha^{m^{n}-\frac{1}{m-1}-\frac{1}{n}}<k \leq\left(\frac{n^{2}+1}{n^{2}}\right)^{1 / m} \alpha^{m^{n}-\frac{1}{m-1}-\frac{1}{n}}\right\} .
$$

Choose $n_{1}$ large enough such that for any $n \geq n_{1}$,

$$
\begin{gathered}
\frac{1}{n}<\frac{1}{2} \log \alpha, \quad \alpha^{-m / n}>1 / 2, \\
\left(\left(\frac{n^{2}+1}{n^{2}}\right)^{1 / m}-1\right) \alpha^{m^{n}-\frac{1}{m-1}-\frac{1}{n}}>1, \\
\frac{1}{4}(m-1) \log \alpha \cdot \alpha^{m^{n}-\frac{1}{m-1}-\frac{1}{n}}>K(n) .
\end{gathered}
$$

For any $n \geq 1$, let

$$
L_{n}=\alpha^{m^{n+n_{1}}-\frac{1}{m-1}-\frac{1}{n+n_{1}}}, \quad M_{n}=\left(\frac{\left(n+n_{1}\right)^{2}+1}{\left(n+n_{1}\right)^{2}}\right)^{1 / m} \alpha^{m^{n+n_{1}-\frac{1}{m-1}-\frac{1}{n+n_{1}}}} .
$$

Note that $e^{x} \geq 1+x$ for any $x \geq 0$. We have, for any $n \geq 1$ and $L_{n}<j \leq M_{n}$,

$$
\begin{aligned}
L_{n+1}-\alpha M_{n}^{m} & =\alpha^{m^{n+n_{1}+1}-\frac{1}{m-1}}\left(\alpha^{-\frac{1}{n+n_{1}+1}}-\frac{\left(n+n_{1}\right)^{2}+1}{\left(n+n_{1}\right)^{2}} \alpha^{-\frac{m}{n+n_{1}}}\right) \\
& =\alpha^{m^{n+n_{1}+1}-\frac{1}{m-1}} \alpha^{-\frac{m}{n+n_{1}}}\left(\alpha^{\frac{m}{n+n_{1}}-\frac{1}{n+n_{1}+1}}-\frac{\left(n+n_{1}\right)^{2}+1}{\left(n+n_{1}\right)^{2}}\right) \\
& \geq \alpha^{m^{n+n_{1}+1}-\frac{1}{m-1}} \alpha^{-\frac{m}{n+n_{1}}}\left(\alpha^{\frac{m-1}{n+n_{1}+1}}-\frac{\left(n+n_{1}\right)^{2}+1}{\left(n+n_{1}\right)^{2}}\right) \\
& \geq \alpha^{m^{n+n_{1}+1}-\frac{1}{m-1}} \alpha^{-\frac{m}{n+n_{1}}}\left(\frac{m-1}{n+n_{1}+1} \log \alpha-\frac{1}{\left(n+n_{1}\right)^{2}}\right) \\
& \geq \frac{1}{2}(m-1) \log \alpha \cdot \alpha^{m^{n+n_{1}+1}-\frac{1}{m-1}} \alpha^{-\frac{m}{n+n_{1}}} \\
& \geq \frac{1}{4}(m-1) \log \alpha \cdot \alpha^{m^{n+n_{1}+1}-\frac{1}{m-1}}>K\left(n+n_{1}\right) .
\end{aligned}
$$

By the definition of $K\left(n+n_{1}\right)$, we have, for any $n \geq 1$ and $L_{n}<j<M_{n}$,

$$
L_{n+1} \geq h_{n}(j)+1 \text {. }
$$

Now just follow the proof of Theorem 1; we omit the details.

By Theorem 5, we get the following corollaries immediately.

COROllary 4. For Sylvester expansion and generalized Cantor product, for any $\alpha \geq 1$, the set

$$
\left\{x \in(0,1]: \lim _{n \rightarrow \infty} d_{n+1}(x) / d_{n}^{2}(x)=\alpha\right\}
$$

is of Hausdorff dimension 1. 
Corollary 5. For Daróczy-Kátai-Birthday expansion, for any $\alpha \geq 1$, the set

$$
\left\{x \in(0,1]: \lim _{n \rightarrow \infty} d_{n+1}(x) / d_{n}^{3}(x)=\alpha\right\}
$$

is of Hausdorff dimension 1.

4. The exceptional set of the uniform distribution modulo 1 . In this section, we always assume $a_{n}(j)=a(j), b_{n}(j)=b(j)$ for any $n \geq 1$. In this case, $h_{n}(j)$ does not depend on $n$. Consider the map $T:(0,1] \rightarrow(0,1]$ defined by

$$
T x=\left(x-\frac{1}{k+1}\right) \frac{b(k)}{a(k)} \quad \text { on }\left(\frac{1}{k+1}, \frac{1}{k}\right] .
$$

Schweiger (see [17, Chapter 11, Theorem 11.1.1]) proved

THEOREM 6. Suppose that $h(k) \geq k-1$. Then for almost every $x$, the sequence $\left(h\left(d_{n}(x)\right) T^{n}(x)\right)_{n \geq 1}$ is uniformly distributed modulo 1 .

Let

$F=\left\{x \in[0,1):\left(h\left(d_{n}(x)\right) T^{n}(x)\right)_{n \geq 1}\right.$ is not uniformly distributed modulo 1$\}$.

THEOREM 7. Suppose that $h(k) \geq k-1$ and $h(k)$ is of order $t$ with $\beta>0$. Then $\operatorname{dim}_{\mathrm{H}} F=1$.

Proof. For any $x \in(0,1]$, let

$$
x=\frac{1}{d_{1}}+\frac{a_{1}}{b_{1}} \frac{1}{d_{2}}+\ldots+\frac{a_{1} a_{2} \ldots a_{n}}{b_{1} b_{2} \ldots b_{n}} \frac{1}{d_{n+1}}+\ldots
$$

be the Oppenheim expansion of $x$. By the algorithm for the Oppenheim expansion, we have for any $n \geq 1$,

$$
T^{n}(x) \in\left(\frac{1}{d_{n+1}(x)}, \frac{1}{d_{n+1}(x)-1}\right) .
$$

This implies

$$
h\left(d_{n}(x)\right) T^{n}(x) \geq \frac{h\left(d_{n}(x)\right)}{d_{n+1}(x)} .
$$

Choose $M>\max (1,1 / \beta)$. We see from Corollary 1 that for any $x \in \bar{A}(M)$, there exists $n(x)$ such that $h\left(d_{n}(x)\right) T^{n}(x) \notin[0,1 /(2 M)]$ for any $n \geq n(x)$, thus $\left(h\left(d_{n}(x)\right) T^{n}(x)\right)_{n \geq 1}$ is not uniformly distributed modulo 1 . This implies $\bar{A}(M) \subset F$. Since $\operatorname{dim}_{\mathrm{H}} \bar{A}(M)=1$, we have $\operatorname{dim}_{\mathrm{H}} F=1$.

Acknowledgements. The author thanks the referee for his (her) valuable suggestions. 


\section{References}

[1] P. Erdős, A. Rényi and P. Szüsz, On Engel's and Sylvester's series, Ann. Univ. Sci. Budapest. Eötvös Sect. Math. 1 (1958), 7-32.

[2] E. B. Escott, Rapid method for extracting square roots, Amer. Math. Monthly 44 (1947), 644-646.

[3] K. J. Falconer, Techniques in Fractal Geometry, Wiley, 1997.

[4] J. Galambos, The ergodic properties of the denominators in the Oppenheim expansion of real numbers into infinite series of rationals, Quart. J. Math. Oxford Ser. (2) 21 (1970), 177-191.

[5] - Further ergodic results on the Oppenheim series, ibid. 25 (1974), 135-141.

[6] - On the speed of convergence of Oppenheim series, Acta Arith. 19 (1971), 335342.

[7] -, Representations of Real Numbers by Infinite Series, Lecture Notes in Math. 502, Springer, 1976.

[8] - , The extension of William's method to the metric theory of general Oppenheim expansions, Studia Sci. Math. Hungar. 26 (1991), 321-327.

[9] - Further metric results on series expansions, Publ. Math. Debrecen 52 (1998), 377-384.

[10] Y. Lacroix, Metric properties for generalized Cantor products, Acta Arith. 63 (1993), $61-77$.

[11] Y. Lacroix and A. Thomas, Number systems and repartition, J. Number Theory 49 (1994), 308-318.

[12] Y. Y. Liu and J. Wu, Hausdorff dimensions in Engel expansions, Acta Arith. 99 (2001), 79-83.

[13] A. Oppenheim, On the representation of real numbers by products of rational numbers, Quart. J. Math. Oxford Ser. 4 (1953), 303-307.

[14] - The representation of real numbers by infinite series of rationals, Acta Arith. 21 (1972), 391-398.

[15] A. Rényi, On Cantor's product, Colloq. Math. 6 (1958), 135-139.

[16] —, A new approach to the theory of Engel's series, Ann. Univ. Sci. Budapest. Eötvös Sect. Math. 5 (1962), 25-32.

[17] F. Schweiger, Ergodic Theory of Fibred Systems and Metric Number Theory, Clarendon Press, Oxford, 1995.

[18] W. Vervaat, Success Epochs in Bernoulli Trials, Math. Center Tracts 42, Mathematisch Centrum, Amsterdam, 1972.

[19] J. Wu, On the distribution of denominators in Sylvester expansions, Bull. London Math. Soc. 34 (2002), 16-20.

Department of Mathematics

Wuhan University

430072 Wuhan

People's Republic of China

E-mail: wujunyu@public.wh.hb.cn

Received on 29.1.2002

and in revised form on 23.5.2002 